\title{
Defective high-affinity thiamine transporter leads to cell death in thiamine-responsive megaloblastic anemia syndrome fibroblasts
}

\author{
Amy R. Stagg, ${ }^{1}$ Judith C. Fleming, ${ }^{1}$ Meghan A. Baker, ${ }^{1}$ \\ Massayuki Sakamoto, ${ }^{2}$ Nadine Cohen, ${ }^{3}$ and Ellis J. Neufeld ${ }^{1}$ \\ ${ }^{1}$ Division of Hematology/Oncology, and \\ ${ }^{2}$ Department of Laboratory Medicine, Children's Hospital, Dana Farber Cancer Institute, \\ and Harvard Medical School, Boston, Massachusetts 02115, USA \\ ${ }^{3}$ Tamkin Molecular Genetics Facility, Technion Faculty of Medicine, Haifa 31096, Israel
}

Address correspondence to: Ellis J. Neufeld, Division of Hematology, Enders 720, 300 Longwood Avenue,

Boston, Massachusetts 02115, USA. Phone: (617) 355-8183; Fax: (617) 734-6791; E-mail: neufeld@a1.tch.harvard.edu

Amy R. Stagg and Judith C. Fleming contributed equally to this work.

Received for publication May 1, 1998, and accepted in revised form January 12, 1999.

\begin{abstract}
We have investigated the cellular pathology of the syndrome called thiamine-responsive megaloblastic anemia (TRMA) with diabetes and deafness. Cultured diploid fibroblasts were grown in thiamine-free medium and dialyzed serum. Normal fibroblasts survived indefinitely without supplemental thiamine, whereas patient cells died in 5-14 days (mean 9.5 days), and heterozygous cells survived for more than 30 days. TRMA fibroblasts were rescued from death with $10-30 \mathrm{nM}$ thiamine (in the range of normal plasma thiamine concentrations). Positive terminal deoxynucleotide transferase-mediated dUTP nick end-labeling (TUNEL) staining suggested that cell death was due to apoptosis. We assessed cellular uptake of $\left[{ }^{3} \mathrm{H}\right]$ thiamine at submicromolar concentrations. Normal fibroblasts exhibited saturable, highaffinity thiamine uptake $\left(K_{\mathrm{m}} 400-550 \mathrm{nM} ; V_{\max } 11 \mathrm{pmol} / \mathrm{min} / 10^{6}\right.$ cells $)$ in addition to a low-affinity unsaturable component. Mutant cells lacked detectable high-affinity uptake. At $30 \mathrm{nM}$ thiamine, the rate of uptake of thiamine by TRMA fibroblasts was 10-fold less than that of wild-type, and cells from obligate heterozygotes had an intermediate phenotype. Transfection of TRMA fibroblasts with the yeast thiamine transporter gene THI10 prevented cell death when cells were grown in the absence of supplemental thiamine. We therefore propose that the primary abnormality in TRMA is absence of a high-affinity thiamine transporter and that low intracellular thiamine concentrations in the mutant cells cause biochemical abnormalities that lead to apoptotic cell death.
\end{abstract}

J. Clin. Invest. 103:723-729 (1999).

\section{Introduction}

The syndrome called thiamine-responsive megaloblastic anemia (TRMA) with diabetes and deafness (OMIM 249270) was first described by Rogers et al. (1). This autosomal recessive disorder with early childhood onset has recently been localized to chromosome 1 , band $1 q 23.23 .3$, in four large, geographically and ethnically distinct kindreds (2). Data from several additional families have sharpened the TRMA interval to a 4-cM region and provided evidence for locus homogeneity (3), suggesting that a mutation in a single gene is responsible for the triad of features that define this disease. More than a dozen case reports have described the clinical manifestations of the disorder in 31 patients (2). In all reported cases, the anemia is corrected by pharmacologic doses of thiamine, although macrocytosis (2) and defects in erythroid differentiation in vitro (4) persist after therapy. The disorder represents a novel form of childhood non-type I diabetes mellitus. No anti-insulin or anti-islet antibodies have been found in patients, and in some cases, pharmacologic doses of thiamine have reduced or eliminated insulin requirements. Hearing loss is irreversible in all patients, although some investigators have reported that thiamine therapy prevented further progression of deafness (5). In addition to the definitive clinical characteristics of TRMA, several patients have had strokelike episodes and/or arrhythmias, and abnormalities of the retina and optic nerve (2).

Normal thiamine homeostasis involves the following steps: (a) uptake of thiamine from the gut, $(b)$ transport to tissues and into cells, $(c)$ conversion of thiamine into the cofactor form thiamine pyrophosphate (TPP) by thiamine pyrophosphokinase (TPK; EC 2.7.6.2), and (d) binding of TPP to apoenzymes. Studies of human intestinal thiamine absorption (6-8) suggest that two uptake pathways exist: transport by a saturable, highaffinity/low-capacity carrier, as well as passive intake by a low-affinity/high-capacity system. Because thiamine concentrations in the intestinal lumen are low, Laforenza et al. (8) propose that most thiamine is absorbed into the body by way of the high-affinity transporter. A similar pattern of thiamine uptake has also been seen in human erythrocytes $(5,9,10)$. Whether the erythroid and intestinal thiamine uptake activities are identical is unknown. After intracellular thiamine is converted to the active cofactor TPP, TPP is incorporated into four known mam- 


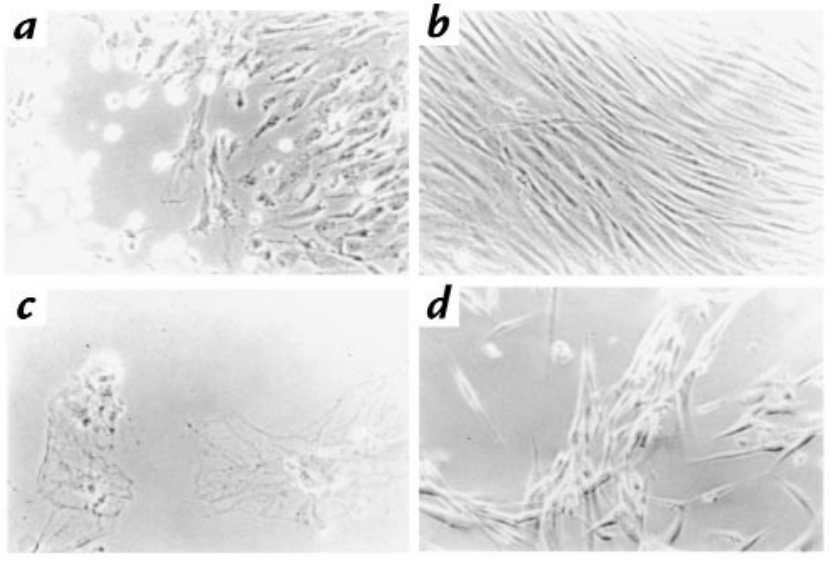

Figure 1

Reversible toxicity in $\mathrm{TRMA}^{-/-}$cells maintained in thiamine-depleted medium. Fibroblasts from a patient with TRMA were grown for 9 days without thiamine $(\boldsymbol{a})$ or with $3 \mathrm{mM}$ supplemental thiamine $(\boldsymbol{b})$. Without thiamine, all cells in thi- medium die by day $10(\boldsymbol{c})$. Addition of $3 \mathrm{mM}$ thiamine at day 9 rescues the remaining viable cells within $24 \mathrm{~h}(\boldsymbol{d}) . \times 20(a$, $b, d) ; \times 40(c)$. Thi-medium, $\alpha$-MEM minus thiamine; TRMA, thiamineresponsive megaloblastic anemia.

malian enzymes: the pentose phosphate shunt enzyme transketolase and three multisubunit, multicofactor enzyme complexes involved in oxidative decarboxylation reactions - pyruvate dehydrogenase, $\alpha$-ketoglutarate dehydrogenase, and branched-chain keto-acid dehydrogenase. The genes encoding the TPP-binding E1 subunits of each of these enzymes have been localized by other investigators and none map to chromosome 1q (2).

Several investigators have proposed a variety of putative defects of thiamine metabolism in patients with TRMA. On the basis of low levels of $\alpha$-ketoglutarate dehydrogenase found in lymphocytes from a patient with TRMA, Abboud et al. (11) proposed that a defective TPP binding site on the enzyme leads to TRMA. Others $(12,13)$ have cited mildly reduced levels of TPK activity as a primary defect. Poggi et al. (5) first noted low TPP content of TRMA erythrocytes ( $\sim 50 \%$ of controls) and postulated that lack of a high-affinity thiamine transporter may be associated with the syndrome $(5,9,10$, 14). The magnitude of the reported defect in thiamine uptake of erythrocytes was subtle, $\sim 60 \%$ of normal. Additionally, these authors suggested that decreased TPK activity contributes to TRMA, as they observed a $20 \%-30 \%$ decrease in TPK activity in TRMA erythrocytes compared with controls.

No mammalian thiamine transporter has been isolated. A thiamine transporter gene from Saccharomyces cerevisiae has been identified $(15,16)$. The THI10 gene encodes a protein with multiple membrane spanning domains, which confers high-affinity thiamine transport in yeast.

In the present study, we demonstrate that fibroblasts from patients with TRMA die in the absence of exogenous thiamine. Using normal or mutant cells, we have explored the thiamine uptake system and the ability of the yeast transporter to correct the lethality in thiaminedepleted cultures. This has enabled us to clarify previous data and to test earlier hypotheses regarding possible defects in TRMA. On the basis of these experiments, we propose a model for the pathogenesis of TRMA in which absence of a very-high-affinity thiamine transporter causes a decrease in cell survival.

\section{Methods}

Patients. Samples were obtained from members of a large, inbred Alaskan kindred reported previously (2). Fibroblasts were obtained from skin biopsies of three patients, several obligate heterozygotes (parents), and non-blood relatives (wild-type). Informed consent was obtained from all subjects and their parents.

Cell culture and cell lines. Diploid fibroblasts were grown from skin samples in thiamine-replete $\alpha$-MEM $(1 \mathrm{mg} / \mathrm{l}$ thiamine; GIBCO BRL, Grand Island, New York, USA), 15\% FCS, 50 U/ml penicillin, $300 \mu \mathrm{g} / \mathrm{ml}$ streptomycin sulfate (GIBCO BRL) in 5\% $\mathrm{CO}_{2}$ at $37^{\circ} \mathrm{C}$. $\alpha$-MEM minus thiamine (thi- medium) with $15 \%$ dialyzed FCS (GIBCO BRL) was used for thiamine starvation. Thiamine depletion was begun after cells were washed three times in PBS and passaged into thi- medium. Experiments shown were performed on two TRMA cell lines (first cousins), two lines from obligate heterozygotes, and two normal controls.

Thiamine uptake assays. $\left[{ }^{3} \mathrm{H}\right]$ thiamine hydrochloride (specific activity $15 \mathrm{Ci} / \mathrm{mmol}$; American Radiolabeled Chemicals Inc., St. Louis, Missouri, USA) was used for uptake assays. Thiamine uptake was assayed in extracellular concentrations ranging from $33 \mathrm{nM}$ to $3 \mathrm{mM}$. Cells were washed with PBS, trypsinized, and plated at a density of $1-2 \times 10^{5}$ cells $/ 35-\mathrm{mm}$ well in $1 \mathrm{ml}$ of thi- medium. Uptake was measured in cells incubated at $37^{\circ} \mathrm{C}$ in thi- medium without FCS. In unlabeled thiamine competition assays, thiamine-HCl (Sigma Chemical Co., St. Louis, Missouri, USA) was added at the appropriate concentration. After cell labeling, cells were washed three times with PBS and harvested with $0.05 \%$ trypsin-EDTA (GIBCO BRL). The amount of cell-associated $\left[{ }^{3} \mathrm{H}\right]$ thiamine was determined by scintillation counting in $5 \mathrm{ml}$ Ecolume scintillation fluid (ICN Radiochemicals Inc., Costa Mesa, California, USA) using a Beckman LS 3801 instrument (Beckman Instrument Inc., Palo Alto, California, USA). Incubation at $0^{\circ} \mathrm{C}$ was used as a control for passive, cell-associated radioactivity.

Assay for $\alpha$-ketoglutarate dehydrogenase. The method was adapted from a pyruvate dehydrogenase assay described by Hansen (17). $\alpha$-ketoglutarate dehydrogenase catalyzes the formation of succinyl CoA from $\left[1-{ }^{14} \mathrm{C}\right] \alpha$-ketoglutarate, resulting in release of ${ }^{14} \mathrm{CO}_{2}$, which is trapped and measured. The mitochondrial fraction (10,000-g pellet of postnuclear supernatant) was incubated with $1 \mu \mathrm{Ci}\left[{ }^{14} \mathrm{C}\right] \alpha$-ketoglutarate (Du Pont NEN Research Products, Boston, Massachusetts, USA) diluted with nonlabeled $\alpha$-ketoglutarate to $1 \mathrm{mM}, 1 \mathrm{mM}$ coenzyme A (lithium salt; Sigma Chemical Co.), $1.6 \mathrm{mM}$ nicotinamide adenine dinucleotide, $40 \mathrm{mM}$ sodium phosphate $(\mathrm{pH} 7.4), 0.5 \mathrm{mM}$ dithiothreitol, and $5 \mathrm{mg} / \mathrm{ml} \mathrm{BSA}$, in the presence or absence of $1 \mathrm{mM}$ TPP. The reaction was carried out in a 5 -cc vial sealed

\section{Table 1}

Concentration of thiamine required to prevent toxicity in TRMA fibroblasts

$0.3 \mathrm{nM} \quad 0.9 \mathrm{nM} \quad 3 \mathrm{nM} \quad 9 \mathrm{nM} \quad 30 \mathrm{nM} \quad 300 \mathrm{nM} \quad 900 \mathrm{nM}$

$\begin{array}{lllllllll}\text { TRMA } & - & - & - & \pm & + & +\end{array}$

Control $\quad+\quad+\quad+\quad+\quad+\quad+\quad+\quad+\quad+$

Cells were washed, trypsinized, and replated in varying thiamine concentrations and then maintained for 30-40 days. Plus sign indicates cell survival, minus sign indicates cell death, and plus over minus indicates morphologic changes without cell death. Normal human plasma contains 18-32 nM free thiamine. Replete MEM contains $>3,000 \mathrm{nM}$. Results are from duplicate wells in three individual experiments. TRMA, thiamine-responsive megaloblastic anemia. 


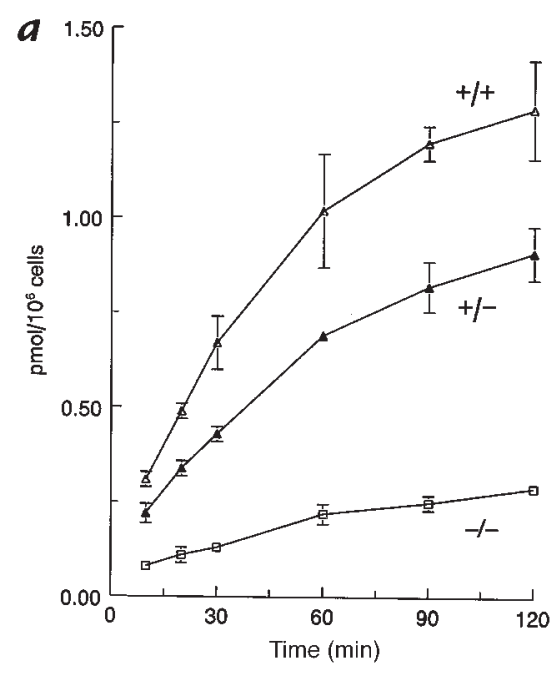

$\boldsymbol{b}$

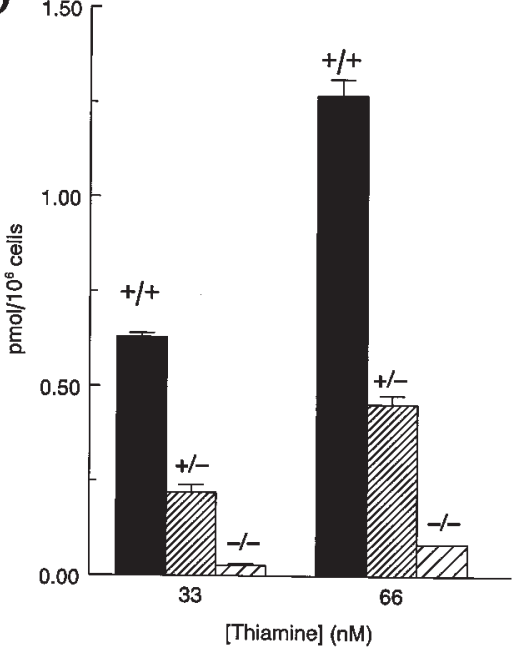

Figure 2

Uptake of $\left[{ }^{3} \mathrm{H}\right]$ thiamine by TRMA fibroblasts. (a) Time course of uptake. Cells were washed and placed in thi- medium 1 day before assay. Uptake was measured for times shown, at $37^{\circ} \mathrm{C}$, in serum-free medium supplemented with $66 \mathrm{nM}$ thiamine as described in Methods (mutant, -/-; heterozygote, +/-; normal, +/+). Means of duplicate wells are given. Values are corrected for cell-associated radioactivity at $0^{\circ} \mathrm{C}$. (b) Defective specific uptake in $\mathrm{TRMA}^{-/-}$ cells, assessed after $30 \mathrm{~min}$ incubation at $37^{\circ} \mathrm{C}$. Specific uptake was calculated by subtracting radioactivity incorporated in the presence of 3 $\mathrm{mM}$ unlabeled thiamine. Means of duplicate wells from one subject of each genotype are represented. Error bars represent ranges for duplicate determinations. with a serum cap, with a hanging basket containing a small piece of filter paper soaked in $2.5 \mathrm{M} \mathrm{NaOH}$, and was quenched after $60 \mathrm{~min}$ with $3 \mathrm{M}$ acetic acid for $1 \mathrm{~h}$ at $37^{\circ} \mathrm{C}$. The filter paper with adsorbed ${ }^{14} \mathrm{CO}_{2}$ was taken from the hanging vessel and counted directly in fluor. The assay was linear with respect to time and protein concentration.

Assay for conversion of thiamine to TPP. TRMA and normal fibroblasts were plated at a density of $10^{6}$ cells $/ 100-\mathrm{mm}$ dish and incubated in thi- medium. The following day, the medium was removed and cells were incubated in thi- medium containing $2.4 \mu \mathrm{M}\left[{ }^{14} \mathrm{C}\right]$ thiamine (specific activity of $24 \mathrm{mCi} / \mathrm{mmol}$; Amersham International, Buckinghamshire, United Kingdom) at $37^{\circ} \mathrm{C}$ for $18 \mathrm{~h}$. Cells were then washed three times with PBS on ice, harvested with $0.05 \%$ trypsin-EDTA, centrifuged, and washed two more times with PBS; an internal standard (unlabeled thiamine, thiamine monophosphate, and TPP) was added; and the protein was removed with $50 \mu \mathrm{l} 5 \% \mathrm{TCA}$. The supernatant was added to $1 \mathrm{ml}$ of $20 \mathrm{mM}$ Tris ( $\mathrm{pH} 8.0$ ). Conversion of thiamine to TPP was analyzed by fast protein liquid chromatography (FPLC; Pharmacia Biotech Inc., Piscataway, New Jersey, USA) on a Mono Q column, and fractions were collected a rate of $1 \mathrm{ml} / \mathrm{min}$ with a set gradient from 0 to $1 \mathrm{M} \mathrm{NaCl}$ in 20 $\mathrm{mM}$ Tris ( $\mathrm{pH} 8.0)$. Free thiamine ran in the void volume, and TPP eluted with $0.35 \mathrm{M} \mathrm{NaCl}$ and TMP at an intermediate position. Internal standard peaks, followed by ultraviolet absorption at $254 \mathrm{~nm}$, showed similar recovery of all three compounds.

Apoptosis TUNEL assay. Apoptosis of TRMA fibroblasts was evaluated by using an in situ cell detection kit (Boehringer Mannheim Biochemicals, Indianapolis, Indiana, USA). Positive cells were visualized using peroxidase substrate enhancer and metal-enhanced DAB substrate (Boehringer Mannheim Biochemicals). TRMA fibroblasts and normal controls were split into thi- medium and grown on glass coverslips. Cells were assayed on 4 consecutive days, beginning on the day when the cell morphology was distinctly different in the mutants. A terminal deoxynucleotide transferase-mediated dUTP nick endlabeling (TUNEL) assay was performed as suggested by the manufacturer, except that the ratio of terminal transferase enzyme solution to label solution was 1:15. Cell were counterstained with $2 \%$ methyl green-pyronin.

Organic acid analysis. Mutant or control cells were plated in 35 $\mathrm{mm}$ dishes and incubated overnight in thi- medium. The medium was changed on day 0 , and then medium was collected from duplicate wells after 1 and 6 days. The cells from each well were counted. Media were stored frozen before analysis. Onemilliliter samples of medium from six-well plates were treated with hydroxylamine $\mathrm{HCl}$ to oximize the ketoacids, then acidified with $\mathrm{HCl}$ and extracted into ethyl acetate, followed by extraction with diethyl ether. Samples were dehydrated with $\mathrm{Na}_{2} \mathrm{SO}_{4}$, and the solvent was evaporated under $\mathrm{N}_{2}$. Organic acids were derivatized with $100 \mu \mathrm{l}$ BSTFA (Alltech Associates Inc., Deerfield, Illinois, USA) and incubated for $10 \mathrm{~min}$ at $60^{\circ} \mathrm{C}$. Aliquots $(1 \mu \mathrm{l})$ were injected for gas chromatography-mass spectrometry analysis on a DB-1 column (dimethylpolysiloxane; Alltech Associates Inc.) in a Hewlett-Packard instrument (Hewlett-Packard, Santa Clarita, California, USA). Peaks at retention times corresponding to the expected compounds were checked by mass spectroscopy against standard spectra libraries. These compounds included lactate, pyruvate, 2ketoisovalerate, 2-keto-3-methylvalerate, 2-ketoisocaproate, and 2-ketoglutarate. As an internal standard for each reaction, $10 \mu \mathrm{g}$ of pentadecanoic acid was added to each medium sample before extraction and derivitization, to control for recovery. Standards for authentic $\alpha$-ketoacids were obtained from Sigma Chemical Co. Results (peak heights) were compared by normalizing to the size of the internal standard peak and corrected for cell number in each dish.

Expression of yeast thiamine transporter gene in TRMA fibroblasts. The yeast thiamine transporter gene THI1O was amplified with Pfu DNA polymerase (Stratagene Inc., La Jolla, California, USA) from total yeast genomic DNA (Promega Corp., Madison, Wisconsin, USA) using primers flanking the open reading frame YRL237w. The primers contained EcoRI restriction sites to aid in subsequent cloning steps. Amplified

\section{Table 2}

Uptake and conversion of $\left[{ }^{14} \mathrm{C}\right]$ thiamine to TPP at steady state by normal and $\mathrm{TRMA}^{-/-}$fibroblasts

\begin{tabular}{|c|c|c|}
\hline Total thiamine & TPP & Conversion (\%) \\
\hline \multicolumn{3}{|c|}{$\mathrm{pmol} / 10^{6}$ cells } \\
\hline 12 & 4.1 & $45 \pm 6$ \\
\hline 9.2 & 5.3 & $58 \pm 7$ \\
\hline
\end{tabular}

Cells were washed and plated in thi- medium overnight before incubation with 2.4 $\mathrm{mM}\left[{ }^{14} \mathrm{C}\right]$ thiamine for $18 \mathrm{~h}$. Cell-associated radioactivity was extracted in 5\% TCA and resolved by FPLC anion exchange chromatography as described in Methods. Determinations are means $\pm \mathrm{SD}$ of triplicate determinations. FPLC, fast protein liquid chromatography; thi-medium, $\alpha$-MEM minus thiamine; TPP, thiamine pyrophosphate. 


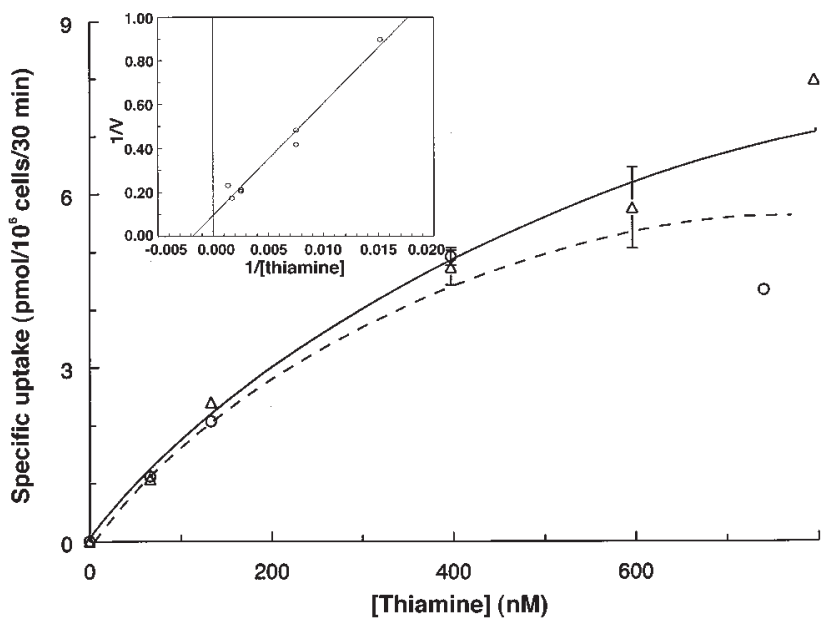

Figure 3

Concentration dependence of high-affinity thiamine uptake in normal fibroblasts. Cells were labeled with $66 \mathrm{nM}\left[{ }^{3} \mathrm{H}\right]$ thiamine diluted with unlabeled thiamine for $30 \mathrm{~min}$ at $37^{\circ} \mathrm{C}$. Values are corrected for nonspecific uptake by subtracting counts associated with the presence of 500-fold excess unlabeled compound. Results are shown for two separate experiments (solid line with triangles and dotted line with circles), done on different days with different wild-type cell lines. Inset: double reciprocal plot; the 800-nM point was eliminated as an outlier. All other data from the rectangular plot were included. The least-squares regression line gives an apparent $K_{\mathrm{m}}$ of $550 \mathrm{nM}$; apparent $V_{\max }$ for uptake is $11 \mathrm{pmol} / 10^{6} \mathrm{cell} / \mathrm{s} / 30$ $\mathrm{min}$. Each point is the mean of duplicate wells \pm range (error bars).

THI10 was cloned into the mammalian expression vector pEF1 $\alpha$-neo and confirmed by restriction and sequence analysis. Fibroblasts from patients with TRMA were cultured as already described here. On the day before transfection, confluent $100-\mathrm{mm}$ tissue culture dishes were split 1:2 and plated in replete medium lacking antibiotics. Lipofectamine Plus Reagent (4 mg; GIBCO BRL) was used for the transfection of either pEF $1 \alpha$-neo/THI10 or pEF $1 \alpha$-neo alone into the TRMA fibroblasts in serum-free medium. Three hours after transfection, medium with serum was added so that the final concentration of FCS was $15 \%$ and thiamine was $3 \mu \mathrm{M}$. Transfected cells were maintained at $37^{\circ} \mathrm{C}$ and $5 \% \mathrm{CO}_{2}$. The medium was replaced $24 \mathrm{~h}$ after transfection with medium containing $300 \mu \mathrm{g} / \mathrm{ml} \mathrm{G} 418$ (GIBCO BRL) to select transformants. Medium was changed every 2-3 days. Three weeks after transfection, G418-resistant cells were replated to be $50 \%-75 \%$ confluent and were maintained in medium with or without thiamine at no more than $90 \%$ confluence.

\section{Results}

Thiamine deprivation of TRMA fibroblasts. Fibroblasts from patients with TRMA and from normal controls were assessed for a thiamine-dependent phenotype by growing cells in thi- medium. After 5-14 days of thiamine starvation, the morphology of TRMA fibroblasts began to change (Fig. 1a). The mean time to abnormal morphology was 9.5 days ( \pm 5 days, $S D$ ) in eight experiments. Cells maintained in thiamine-replete medium remained indistinguishable from wild-type (Fig. 1b). Within 1-3 days of morphological evidence of toxicity, cells in thimedium detached or died on the plates (Fig. 1c). However, addition of thiamine $(3 \mu \mathrm{M})$ to TRMA fibroblasts when adverse changes first became evident rescued the remaining cells (Fig. 1d). Rescued cells reverted to normal morphology within 24 hours and could then be grown as before thiamine starvation. Normal fibroblasts in thi- medium showed some decrease in growth rate but did not die under these conditions (experiments terminated at 40 days). Heterozygous cells died after 30 and 32 days on two occasions. These experiments suggest that TRMA fibroblasts are more sensitive to thiamine starvation and can be distinguished from normal fibroblasts by their requirement for exogenous thiamine. We postulate that normal cells survive because of the trace amounts of vitamin (below fluorometric assay) remaining in the dialyzed serum.

The concentration of thiamine necessary to sustain TRMA fibroblasts was investigated by growing cells in medium with thiamine concentrations ranging from 0 to $900 \mathrm{nM}$. Results are shown in Table 1 . Nine nanomolars of thiamine rescued mutant cells to a variable degree, whereas $30 \mathrm{nM}$ vitamin always rescued completely. This concentration is approximately that in plasma and is 100-fold less than the amount in replete tissue culture media $(\geq 3 \mu \mathrm{M})$. We concluded that the defect in TRMA cells might be observable only at extremely low thiamine concentrations.

$\left[{ }^{3} \mathrm{H}\right]$ thiamine uptake in normal, heterozygote, and TRMA fibroblasts. To understand the mechanism of TRMA fibroblast sensitivity to thiamine starvation, the cellular uptake of thiamine was investigated at nanomolar concentrations. $\left[{ }^{3} \mathrm{H}\right]$ thiamine of high specific activity $(15 \mathrm{Ci} / \mathrm{mmol}$, $\sim 15,000 \mathrm{cpm} / \mathrm{pmol}$ ) was used to allow detection of uptake at these low concentrations. Fig. $2 a$ depicts the results of a representative time course experiment. Fibroblasts were incubated in $66 \mathrm{nM}\left[{ }^{3} \mathrm{H}\right]$ thiamine over a period of two hours. Values were corrected for cell-associated counts in cells incubated in an ice bath. These $0^{\circ} \mathrm{C}$ controls were always $<0.1 \mathrm{pmol} / 10^{6}$ cells. However, these results are not corrected for nonspecific, or unsaturable, uptake. Uptake was approximately linear for 30 minutes and approached a plateau by approximately two hours in serum-free medium. Subsequent assays were done at 30 minutes. As demonstrated by this experiment, mutant fibroblasts show a significant defect in thiamine uptake, and heterozygous cells are intermediate in degree of uptake.

We next assessed uptake at varying concentrations of thiamine in 30-minute assays. A substantial difference between mutant and normal thiamine uptake was observed at the lowest concentrations tested (Fig. 2b). These results are corrected for nonspecific uptake by subtracting counts obtained in the presence of $3 \mathrm{mM}$ unlabeled thiamine (50,000 to 100,000-fold excess). The mutants exhibit $~ 5 \%$ of the wild-type uptake at 33 and 66 $\mathrm{nM}$ thiamine under these conditions. This is consistent with either a severe defect or absence of a high-affinity transporter. Laforenza et al. (18) have observed a pH-sensitive thiamine transport system in rat intestine that is augmented by omeprazole. The fibroblast high-affinity uptake system is apparently unaffected by omeprazole or $\mathrm{pH}$ changes in the 6.5-8.0 range (data not shown).

Kinetic properties of high-affinity thiamine transport by fibroblasts. Pilot studies suggested that thiamine uptake became unsaturable at concentrations $<1 \mu \mathrm{M}$. We therefore performed detailed analysis of uptake by normal fibroblasts 
Table 3

Organic acid analysis of medium from TRMA and control fibroblasts in the thi- medium

\begin{tabular}{|c|c|c|c|c|c|}
\hline \multirow{3}{*}{$\begin{array}{l}\text { Conditions } \\
\text { Cell number per dish } \\
\text { at harvest day }\end{array}$} & \multirow[t]{2}{*}{ Medium alone } & \multicolumn{2}{|c|}{ Day 1} & \multicolumn{2}{|c|}{ Day 6} \\
\hline & & Mutant & Control & Mutant & Control \\
\hline & & $4.9 \times 10^{5}$ & $2.1 \times 10^{5}$ & $4.1 \times 10^{5}$ & $7.0 \times 10^{5}$ \\
\hline $\begin{array}{l}\text { Internal standard } \\
\text { (integrator units) }\end{array}$ & 2.85 & 1.87 & 3.4 & 2.46 & 2.18 \\
\hline \multicolumn{6}{|c|}{ Organic acid content (integrator units per million cells) } \\
\hline Lactate & 0 & 10.2 & 7 & 16.3 & 11.0 \\
\hline Pyruvate & 0.38 & 1.07 & 0.63 & 2.47 & 0.41 \\
\hline 2-ketoisovalerate & 0 & 0.07 & 0 & 2.14 & 0 \\
\hline 2-keto-3-methylvalerate & 0 & 0.11 & 0.1 & 0.24 & 0.05 \\
\hline 2-ketoisocaproate & 0 & 0.49 & 0.38 & 0.88 & 0.15 \\
\hline 2-ketoglutarate & 0 & 0 & 0 & 0.14 & 0.06 \\
\hline
\end{tabular}

Media were collected from wild-type and mutant cells on days 1 and 6 after switch ing to thiamine-depleted conditions and were analyzed by gas chromatography-mass spectrometry analysis as described in Methods. Thi- medium (including dialyzed serum) was also analyzed "medium alone." Cells from duplicate wells were counted on the day of harvest, and an internal standard was added to each sample before extraction and derivatization to control for recovery.

in the nanomolar range as shown in Fig. 3. The properties of uptake by growing fibroblasts proved highly reproducible from experiment to experiment and among cell lines from the different normal and mutant cell lines tested. We analyzed only specific, or saturable, uptake by subtracting counts associated with cells in 500-fold excess of unlabeled thiamine. Under these conditions, LineweaverBurke analysis revealed a saturable process with apparent $K_{\mathrm{m}}$ values from 400 to $550 \mathrm{nM}$, and a maximum transport rate of $11 \mathrm{pmol} / 10^{6} \mathrm{cells} / 30 \mathrm{~min}$. We were unable to detect a saturable component in the TRMA fibroblasts in several attempts, although a small amount of residual transport activity could still be present. However, the unsaturable component of thiamine uptake at thiamine concentrations $>1 \mu \mathrm{M}$ was essentially the same in TRMA as in wild-type cells (not shown).

Assays of other candidate enzymes for TRMA. We also examined the function of reported candidate genes, $\alpha$ ketoglutarate dehydrogenase and TPK. We assayed $\alpha$ ketoglutarate dehydrogenase in mitochondrial pellets of fibroblasts from two patients with TRMA or normal controls, as described in Methods, by measuring the coenzyme A-dependent release of ${ }^{14} \mathrm{CO}_{2}$ from $\left[1-{ }^{14} \mathrm{C}\right] \alpha$ ketoglutarate. Results expressed as $\mathrm{nmol} \mathrm{CO}_{2}$ release/mg protein $/ \mathrm{h}$ (mean of duplicate determinations \pm range) were as follows: control, $250( \pm 42)$; TRMA patient no. 1 , 144 ( \pm 39$)$; TRMA patient no. $2,273( \pm 13)$. The differences were not statistically significant and indicated that this enzyme is not defective in the TRMA fibroblasts.

We next measured conversion of thiamine to TPP, as an indirect measurement of TPK activity in vivo, in overnight incubation of mutant or wild-type cells as described in Methods. We found no defect in conversion of thiamine to TPP. Indeed, the degree of conversion of radiolabeled thiamine to TPP (Table 2) was consistently greater in the mutants $(58 \% \pm 7 \%$ vs. $45 \% \pm 6 \%$; mean \pm SD, $P=0.043$ by two-tailed $t$ test). We speculate that the difference is due to smaller intracellular pools of unlabeled thiamine in the mutant compared with the wild-type cells during these experiments. Thiamine monophosphate was a minor product in both wild-type and mutant cells.

Apoptosis assay. Once the phenotype of TRMA fibroblasts in thi- medium became apparent, the mechanism of cell death was investigated. Using the TUNEL assay for fragmented genomic DNA, TRMA and control fibroblasts depleted of thiamine were assayed (Fig. 4). Cells were assayed on day 10 of incubation in thi- medium. Both normal and TRMA fibroblasts are TUNEL negative when grown in thiamine-replete media (Fig. 4, $a$ and $b$, respectively). Normal fibroblasts remain negative for TUNEL staining in thi- medium (Fig. 4c). However, as shown in Fig. 4d, TRMA cells become TUNEL positive as the morphology becomes abnormal. These results suggest that TRMA fibroblasts die by programmed cell death. We were unable to detect a DNA "laddering" pattern of apoptosis in dishes of TRMA cells approaching cell death in thimedium. We postulate that only a small number of cells undergo death at any given time. It is also possible that the positive TUNEL staining represents a mechanism for DNA fragmentation other than apoptosis.

Organic acid accumulation in medium of TRMA cells subjected to thiamine starvation. We postulated that the thiamine uptake defect in TRMA cells should lead to more rapid appearance of a thiamine-depleted biochemical phenotype than wild-type cells. Table 3 shows the results of organic acid analysis of medium from TRMA and control cells after one and six days of thiamine starvation. Compared with thi- medium alone, both mutant and wild-type medium show very little increase of the critical organic acids on day 1 . By day 6 , however, all of these compounds are significantly higher in the mutant cells. This implies lower internal stores of TPP in the mutant cells within a few days, despite the very high concentrations of thiamine in standard medium. Although pyruvate is present in the medium as a supplement, it accumulates severalfold in the mutant cells by day 6 . Lactate rose in both cell lines.

Rescue of thiamine-dependent lethality in TRMA fibroblasts by S. cerevisiae THI10 gene. On the basis of the apparent deficiency of a thiamine transporter in TRMA cells, we tested the ability of S. cerevisiae THI10 gene (thiamine transporter) to allow survival of mutant fibroblasts in thiamine-depleted conditions. The THI10 gene was cloned into expression vector pEF1 $\alpha$-neo as described in Methods and was stably transfected into TRMA cells. As shown in Fig. 5, THI10-transfected cells, but not vector-only controls, were able to survive for more than six weeks in thi- medium. Vector-only controls died after 13 days in thi- medium, and the experiment was terminated after 44 days. These results strongly support the hypothesis that the primary TRMA defect is a thiamine transporter. These results do not prove that the transport defect is across the plasma membrane (as opposed to an internal membrane), but we suspect that this is the case based on the uptake assays described.

\section{Discussion}

We have demonstrated that TRMA fibroblasts are uniquely sensitive to thiamine depletion. Cells die after several days in thi- medium, with DNA fragmen- 

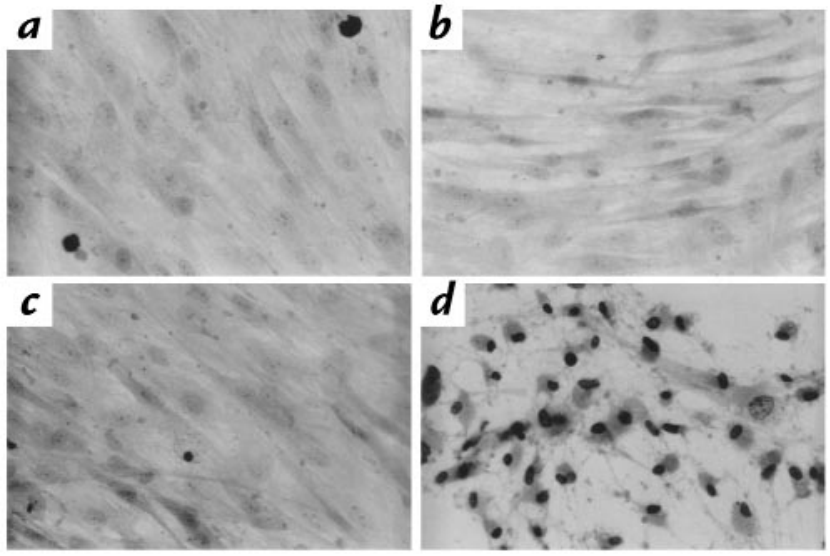

Figure 4

Apoptosis of fibroblasts subjected to thi- medium. TUNEL stain was performed as described in Methods. Normal ( $\boldsymbol{a}$ and $\boldsymbol{b}$ ) or TRMA mutant cells ( $\boldsymbol{c}$ and $\boldsymbol{d}$ ) were incubated for 10 days in the presence ( $a$ and $c$ ) or absence ( $b$ and $d$ ) of thiamine. (a) Wild-type cells, thiamine-replete. ( $b$ ) Wild-type cells, thiamine-depleted. (c) TRMA mutant cells with thiamine. (d) TRMA fibroblasts, thiamine-depleted. $\times 40$. TUNEL, deoxynucleotide transferase-mediated dUTP nick end-labeling.

tation as detected by TUNEL assay consistent with apoptosis or programmed cell death. To our knowledge, this is the first report of a genetic disorder of intermediary metabolism causing apoptosis. Labeling studies using $\left[{ }^{3} \mathrm{H}\right]$ thiamine of high specific activity demonstrate that TRMA fibroblasts take up 5\%-10\% of wild-type amounts of thiamine at nanomolar concentrations. Nonspecific (unsaturable) thiamine uptake is not impaired in the mutants. Thus, in standard tissue culture medium $(3 \mu \mathrm{M}$ thiamine plus the rich supply in serum), the metabolic defect is obscured, whereas in thi- medium, characteristic organic acids accumulate faster in TRMA cells than in normal controls. Our results support the hypothesis that the primary defect in TRMA is a recessive mutation in the gene encoding a high-affinity thiamine transporter. Rescue of the lethal phenotype by a known yeast transporter provides additional support for this hypothesis.

Rindi and colleagues $(9,10)$ have previously defined a subtle defect of thiamine uptake in erythrocytes of patients with TRMA, $\sim 60 \%$ of control. There are at least two possible explanations for the differences observed between their studies and the present work, in which we have found greater than 10 -fold reduction in $\left[{ }^{3} \mathrm{H}\right]$ thiamine uptake at low concentrations. First, the erythrocyte and fibroblast high-affinity systems might be similar or identical, but experimental variables allow the defect to be more readily discerned in the present fibroblast studies. For example, we have used thiamine of specific activity 20 -fold higher than that used in previous studies (15 vs. $0.75 \mathrm{Ci} / \mathrm{mmol}$; refs. 9, 10). This facilitates assays at low substrate concentrations, at which differences between mutant and normal cells are greatest (Fig. 2). The $K_{\mathrm{m}}$ of the high-affinity system described here, $\sim 0.5 \mu \mathrm{M}$, is at the lower end of concentrations studied by Rindi et al. (9). Second, there may be more than one specific thiamine uptake system, oper- ating together or individually in different cell types. If erythrocytes normally have two systems, and fibroblasts only one, the degree of abnormality would appear more severe in the fibroblasts. The current data do not distinguish these possibilities.

Cell death by apparent apoptosis in severely thiaminedepleted TRMA fibroblasts raises several questions about the pathogenesis of the disease. First, why are patients with TRMA not severely ill without pharmacologic thiamine therapy? With a single exception (19), subjects with TRMA have not had symptoms suggestive of severe tissue thiamine deficiency, and they have not had organic aciduria $(5,11)$. Red blood cell transketolase is also normal. We find that $10-30 \mathrm{nM}$ thiamine is enough to rescue fibroblasts (Table 1) and that thiamine depletion does lead to organic acidosis in the cells from patients after a few days of thiamine depletion (Table 3). Normal plasma levels of free thiamine are on the order of $18-33 \mathrm{nM}(5,13)$. This implies that patients with TRMA on an adequate diet without pharmacologic supplementation are metabolically stable, with thiamine uptake solely by the low-affinity system.

Second, why is the deafness in TRMA progressive, whereas the anemia, and to some degree the diabetes, are reversible? We postulate that the thiamine requirement of cochlea or acoustic nerve cells is substantially higher than that of fibroblasts, which have very low energy requirements compared with neurons, myocytes, and other cell types. (We have not examined this phenomenon in other TRMA cell types.) If so, even pharmacologic doses of enteral thiamine may not prevent a thiaminedeficient phenotype in cell types with high energy usage at all times. On the basis of our observations in fibroblasts, we propose that this would lead to occasional cell death in the most sensitive tissues. This, in turn, may result in optic atrophy and progressive deafness. Bone
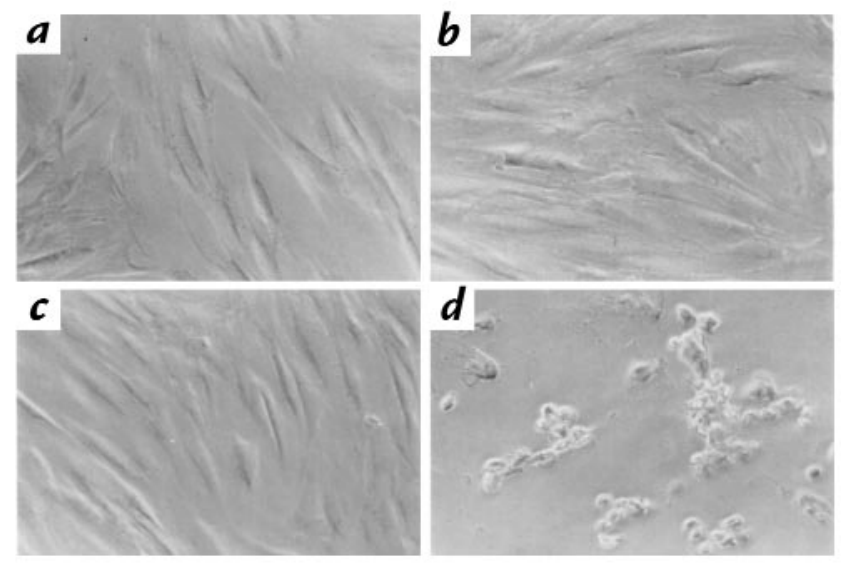

\section{Figure 5}

Complementation of TRMA fibroblasts defects with yeast thiamine transporter gene THI10. Cells transfected with the THI1O gene and vector alone were grown in the presence or absence of thiamine as described in Methods. TRMA fibroblasts transfected with vector alone and grown in thi- medium died after 13 days. The experiment was terminated after 44 days. (a) TRMA fibroblasts transfected with THI10 gene, thiaminereplete. (b) TRMA fibroblasts transfected with vector alone, thiaminereplete. (c) TRMA fibroblasts transfected with THI10 gene, thi- medium. (d) TRMA fibroblasts transfected with vector alone, thi- medium. 
marrow, in contrast, may be less sensitive to this effect for two reasons. First, as long as the pluripotent hematopoietic stem cells are not sensitive to cell death, the erythroid lineage can be repopulated when patients are thiamine replete. Persistent macrocytosis (2) and poor erythroid colony growth in vitro (4) suggest that even thiamine-replete erythroid progenitors are somewhat sensitive to the thiamine transport defect. Diabetes in TRMA patients has been variably reversible with thiamine. It is reasonable to conclude that the beta cells of the pancreas, or the target tissues, are of intermediate sensitivity to cellular thiamine deficiency. Other tissues, notably liver and muscles, which would be responsible for organic acidemia in severe thiamine deficiency, must be resistant to cell toxicity in the ambient thiamine concentration found in untreated patients with TRMA.

It is conceivable that deafness in patients with TRMA could be prevented by parenteral, early (even prenatal) thiamine therapy. Laforenza and colleagues (8) have recently reported a thiamine uptake system from human intestinal biopsies (maximum rate in duodenum but present throughout the gut), with apparent $K_{\mathrm{m}} \sim 4 \mu \mathrm{M}$. Whether this system is due to the same gene product defective in TRMA remains to be determined. The transport across the intestinal epithelium into the abluminal space was not measured. If either of these processes depends on the TRMA gene, it is possible that even pharmacologic doses of oral thiamine cannot provide supranormal levels of the vitamin in the blood and tissues. For cells with high energy requirements, this may not be sufficient in TRMA. We propose that if transintestinal transport is also defective in TRMA, patients may do substantially better if treated from birth (or even prenatally) with parenteral thiamine.

It is not yet clear how the vitamin transport defect in TRMA is linked to programmed cell death. Matsushima et al. (20) recently reported apoptotic cell death in the thalamus of thiamine-deficient rats. This suggests that the phenomenon is not limited to TRMA. However, it does not clarify the relationship between cellular thiamine deficiency and activation of apoptosis pathways. Fibroblasts in thi- medium release organic acids expected from defects in the TPP requiring enzymes, i.e., branched chain $\alpha$-ketoacids and pyruvate (Table 3 ). This suggests that thiamine deprivation can lead to dysfunction in the Krebs cycle, shutting down mitochondrial energy production. We speculate that TRMA cells in thi- medium will be relatively more impaired at these steps than normal fibroblasts. Mitochondrial changes, in turn, may play a pivotal role in activation of apoptosis. A number of possible mechanisms have been proposed (reviewed in ref. 21). Mitochondrial dysfunction might lead to leakage of caspase activators, cytochrome $\mathrm{C}$, or apoptosis-inducing factor, for example. It will be of interest in the future to examine whether TRMA cells from other kindreds exhibit the same uptake kinetics and thiamine-dependent survival as cells from the Alaskan patients tested here. Independent mutations have apparently arisen on different haplotypes in each TRMA family $(2,3)$, and it is possible that differ- ences in phenotype will correlate with different genotypes. In light of our present results, we believe that a candidate gene for TRMA would be a thiamine transporter, responsible for vitamin uptake at low extracellular thiamine concentration. Database homology searches have not yielded such a gene. Isolation of the TRMA gene will facilitate further studies of this unique biochemical disorder.

\section{Acknowledgments}

We express our gratitude to the patients and their families and to Donna Fenske (State of Alaska Division of Public Health, Homer, Alaska, USA). Laura Noriega assisted with thiaminedepletion experiments. We thank Mark Fleming for advice and thoughtful comments. This work was supported by National Institutes of Health grants HL-49196 and HL-07574. Initial portions of this work were supported by a grant from the Charles H. Hood Foundation (to E.J. Neufeld).

1. Rogers, L.E., Porter, F.S., and Sidbury, J.B.J. 1969. Thiamine-responsive megaloblastic anemia. J. Pediatr. 74:494-504.

2. Neufeld, E.J., et al. 1997. Localization of the gene for thiamine-responsive megaloblastic anemia syndrome on the long arm of chromosome 1 by homozygosity mapping. Am. J. Hum. Genet. 61:1335-1341.

3. Raz, T., et al. 1998. Refined mapping of the gene for thiamine-responsive megaloblastic anemia syndrome in a $4 \mathrm{cM}$ region and evidence for genetic homogeneity. Hum. Genet. 103:455-461.

4. Rotoli, B., Poggi, V., De Renzo, A., and Robledo, R. 1986. In vitro addition of thiamin does not restore BFU-E growth in thiamin-responsive anemia syndrome. Haematologica. 71:441-443.

5. Poggi, V., et al. 1984. Thiamin-responsive megaloblastic anaemia: a disorder of thiamin transport? J. Inherit. Metab. Dis. 7:153-154.

6. Rindi, G., and Ferrari, G. 1977. Thiamine transport by human intestine in vitro. Experientia. 33:211-213.

7. Hayumpa, A.M., Jr., et al. 1982. Dual system of intestinal thiamine transport in humans. J. Lab. Clin. Med. 99:701-708.

8. Laforenza, U., et al. 1997. Thiamine uptake in human intestinal biopsy specimens, including observations from a patient with acute thiamine deficiency. Am. J. Clin. Nutr. 66:320-326.

9. Rindi, G., et al. 1992. Thiamine transport by erythrocytes and ghosts in thiamine-responsive megaloblastic anaemia. J. Inherit. Metab. Dis. 15:231-242.

10. Rindi, G., et al. 1994. Further studies on erythrocyte thiamin transport and phosphorylation in seven patients with thiamin-responsive megaloblastic anaemia. J. Inherit. Metab. Dis. 17:667-677.

11. Abboud, M.R., Alexander, D., and Najjar, S.S. 1985. Diabetes mellitus, thiamine-dependent megaloblastic anemia, and sensorineural deafness associated with deficient alpha-ketoglutarate dehydrogenase activity. $J$. Pediatr. 107:537-541.

12. Borgna-Pignatti, C., Marradi, P., Pinelli, L., Monetti, N., and Patrini, C. 1989. Thiamine-responsive anemia in DIDMOAD syndrome. J. Pediatr. 114:405-410.

13. Grill, J., et al. 1991. Thiamine responsive anemia: report of a new case associated with a thiamine pyrophosphokinase deficiency. Nowv. Rev. Fr. Hematol. 33:543-544.

14. Poggi, V., et al. 1989. Studies on thiamine metabolism in thiamineresponsive megaloblastic anaemia. Eur. J. Pediatr. 148:307-311.

15. Enjo, F., Nosaka, K., Ogata, M., Iwashima, A., and Nishimura, H. 1997. Isolation and characterization of a thiamin transport gene, THI10, from Saccharomyces cerevisiae. J. Biol. Chem. 272:19165-19170.

16. Singleton, C.K. 1997. Identification and characterization of the thiamine transporter gene of Saccharomyces cerevisiae. Gene. 199:111-121.

17. Hansen, T.L. 1982. Determination of pyruvate dehydrogenase in cultured human fibroblasts and amniotic fluid cells. Clin. Chim. Acta. 123:45-50.

18. Laforenza, U., Orsenigo, M.N., and Rindi, G. 1998. A thiamine/H+ antiport mechanism for thiamine entry into brush border membrane vesicles from rat small intestine. J. Membr. Biol. 161:151-161.

19. Mandel, H., Berant, M., Hazani, A., and Naveh, Y. 1984. Thiaminedependent beriberi in the "thiamine-responsive anemia syndrome." $N$. Engl. J. Med. 311:836-838.

20. Matsushima, K., MacManus, J.P., and Hakim, A.M. 1997. Apoptosis is restricted to the thalamus in thiamine-deficient rats. Neuroreport. 8:867-870.

21. Mignotte, B., and Vayssiere, J.L. 1998. Mitochondria and apoptosis. Eur. J. Biochem. 252:1-15. 